\title{
Recognition of bilaterally presented words varying in concreteness and frequency: Lateral dominance or sequential processing?
}

\author{
HOWARD B. ORENSTEIN and WALTER B. MEIGHAN \\ Western Maryland College, Westminster, Maryland 21157
}

\begin{abstract}
Words presented in the left visual field were more easily recognized than words presented in the right visual field. This effect was independent of frequency and concreteness. While these results contradict those reported by Ellis and Shepherd (1974), they are in accord with the results of a majority of studies using bilateral stimulus presentation. The discussion stressed the importance of learned responses in perceptual processing and cautioned against attributing functional distinctions to the cerebral hemispheres.
\end{abstract}

Using bilateral presentation across the visual field and a nonordered report, Ellis and Shepherd (1974) confirmed one of their predictions that words presented in the right visual field should yield better recognition than words appearing in the left visual field. They cite an explanation appearing in White (1969; Kimura, 1961) to support their prediction. Interestingly, the bulk of research which White reports using recognition accuracy as the dependent measure has shown the opposite effect, i.e., left visual field superiority. Such left visual field dominance has often been interpreted in terms of ingrained reading habits. Perhaps a more fruitful approach would have been to present visual stimuli bilaterally and use reaction time as the dependent variable. This research technique has more clearly shown hemispheric dominance effects (Gazzaniga, 1972) than the accuracy method. At any rate, in his review of laterality differences, White (1969; see also Paivio, 1971) concluded, in contrast to the findings of Ellis and Shepherd, that (across all types of stimuli) "it has been reasonably well demonstrated that hemifield interacts with presentation to realize a left visual hemifield superiority in the case of bilateral stimulus presentation (p. 403)."

Ellis and Shepherd also confirmed a second prediction concerning superior recognition of concrete, as opposed to abstract, words falling in the left visual field. The basis for this prediction rests on two studies in which the procedures are not explicitly described. In one study, Gazzaniga (1970) found that some split-brain patients were able to recognize only "noun-object words" (not verbs) presented in the left visual field. Presumably, these nouns were highly concrete, but this is not specified in the report. In the

Requests for reprints should be sent to Dr. Howard B. Orenstein, Department of Psychology, Western Maryland College, Westminster. Maryland 21157. Donald A. Schumsky sponsors this paper and takes full editorial responsibility for its content. other study, Marshall (1973), who used normal subjects, found no differential recognition between nouns and verbs presented in the left visual field. However, Marshall did not specify whether he used abstract or concrete nouns. He concluded that the right hemisphere more easily recognized highfrequency, rather than low-frequency, language elements. Although Ellis and Shepherd varied word frequency, they did not present an analysis of errors in terms of any of the possible interactions between visual field, concreteness, and word frequency.

As a result of these problems in analysis and interpretation, the present study was performed in an attempt to replicate the findings reported by Ellis and Shepherd and to clarify the role of concreteness and frequency in recognition of bilaterally presented words.

\section{METHOD}

The procedure was essentially the same as that used by Ellis and Shepherd (1974). Each of 20 concrete words "matched" an abstract word in length (four or five letters) and in Thorndike-Lorge (1944) frequency count. Ellis and Shepherd did not specify the nature of the frequencies for the words they used. However, in the present study, eight of the matched pairs were of high frequency (A or AA) while 12 pairs were of medium-low frequency (with a range of 1-32 per million). Two balanced sets of stimuli were constructed so that each pair of words occurred once in each set but in a reversed position (i.e., if the concrete member of the pair was on the left in one set, it would be on the right side in the other set).

Each subject fixated with both eyes on a small green dot centered on a darkened screen of a projection (rather than look-in) tachistoscope. The end of the left-side word and the beginning of the right-side word were equidistant from the center of fixation (1.35 deg rather than the $1.25 \mathrm{deg}$ used by Ellis \& Shepherd). When ready, the subject pressed a button and two words, each either $1.8 \mathrm{deg}$ or $2.3 \mathrm{deg}$ (depending on length), were exposed for $150 \mathrm{msec}$, followed by the darkened screen. After each paired presentation, the subject was asked to report the words seen in any order he wished, or, if unable to do so, to report any letters he could from each visual field. The seven female and five male subjects were volunteers from Western Maryland College. They were all right-handed and wore no glasses. 


\section{RESULTS}

Because of the unequal number of high- and medium-low frequency words, the whole-word errors for each subject (as a function of visual field, word type. and frequency) were converted to percentages (see means in Table 1). Each of these percentages was then subjected to an arcsin transformation (Snedecor. 1956). Since each subject was exposed to all treatments. a three-factor analysis of variance, with repeated measures on all factors, was performed. The analysis of transformed error percentages revealed that the words appearing in the left visual field, as opposed to right visual field, were more easily recognized, $F(1.11)=9.02, p<.01$. However, none of the remaining main effects nor interactions were significant.

\section{DISCUSSION}

It is clear that the present results, while in direct contrast to those reported by Ellis and Shepherd (1974), are in accord with the majority of evidence concerning verbal material presented bilaterally (see Paivio, 1971; White, 1969). One can only speculate about causes of this discrepancy. Although the words used in the present study were probably different from those used by Ellis and Shepherd, they still were selected from the same source (Paivio et al., 1968) and with similar constraints. Thus, it is difficult to imagine that this variable played a critical role in the discrepancy. It is possible, however, that Ellis and Shepherd's subjects were primarily what Harcum and Filion (1963) termed "mixedsuperior," and those in the present experiment were primarily "left-superior." In Harcum's work, the latter type of subject showed repeated superior recognition for letters in the left visual field regardless of the six experimental conditions. The former showed right visual field superiority for four out of six conditions, and left visual field superiority for the remaining two conditions.

The present results appear to contradict those reported by McKeever (1971). He found right visual field superiority using bilateral presentation of four-letter words. However, he used a stimulus exposure time $(20 \mathrm{msec})$ which was nearly eight times as fast as in the present study. As White (1969) suggests, such a brief (threshold) exposure probably does not permit perception and/or storage of the entire stimulus field. Under these circumstances, the right visual field is more readily scanned as defined by reading habits. Thus, the word in the right visual field is processed at the cost of the word in the left visual field.

To conclude, the present results generally can be interpreted in terms of motor responses acquired while learning to read. It is difficult to know, however, whether the subjects were paying attention to one side of the stimulus array, or whether they had an inclination to report words from left to right. Although "free-report" instructions were used, it is interesting to note that some of the subjects in this study actually began their reports by first saying. "The left word was . . ." As Paivio (1971) aptly
Table 1

Percentage Error as a Function of Visual Field, Word Type, and Word Frequency

\begin{tabular}{|c|c|c|c|c|}
\hline \multirow{3}{*}{$\begin{array}{c}\text { Word } \\
\text { Frequency }\end{array}$} & \multicolumn{2}{|c|}{ Left Visual Field } & \multicolumn{2}{|c|}{ Right Visual Field } \\
\hline & \multicolumn{2}{|c|}{ Word Type } & \multicolumn{2}{|c|}{ Word Type } \\
\hline & Concrete & Abstract & Concrete & Abstract \\
\hline High & .42 & .54 & .58 & .67 \\
\hline Medium-Low & .46 & .50 & .79 & .74 \\
\hline
\end{tabular}

concludes, the left-to-right directional predilection may indicate the learned order of verbal processing which exerts directional control over the postexposure scanning of a stimulus display. Therefore, insofar as suprathreshold bilateral stimulus presentation is concerned, the concept of lateral cerebral dominance may be only an inappropriate label applied to a group of factors related to sequential aspects of perceptual processing.

\section{REFERENCES}

Ellis. H. D.. \& Shepherd. J. W. Recognition of abstract and concrete words presented in left and right visual fields. Journal of Experimental Psychology. 1974. 103. 1035-1036.

Gazzaniga. M. S. The bisected brain. New York: AppletonCentury-Crofts. 1970.

Gazzaniga. M. S. One brain-two minds? American Scientist. 1972. 60. $311-317$.

HaRcly. E. R.. \& Filion. R. D. L. Effects of stimulus reversals on lateral dominance in word recognition. Perceptual and Motor Skills. 1963. 17. 779-794.

Kimura. D. Cerebral dominance and the perception of verbal stimuli. Canadian Journal of Psychology. 1961. 15. 166-171.

MARshall. J. C. Language. learning and laterality. In R. A. Hinde \& J. Stevenson-Hinde (Eds.). Constraints on learning. London: Academic Press. 1973.

MCKEEVER. W. F. Lateral word recognition effects of unilateral and bilateral presentation. asynchrony of bilateral presentation and forced order of report. Quarterh Journal of Experimental Psychology. 1971. 23. 410-416.

PAIvio. A. Imagen and verbal processes. New York: Holt. Rinehart. \& Winston, 1971.

Paivio. A.. Yuille. J. C.. \& Madigan, S. A. Concreteness. imagery, and meaningfulness values of 925 nouns. Journal of Experimental Psychology Monograph Supplement, 1968, 76 (1. Pt. 2).

SNEDECOR. G. W. Statistical methods. Ames, Iowa: The Iowa State University Press. 1956.

THORNDIKE, E. L.. \& LORGE, I. The teacher's wordbook of 30,000 words. New York: Columbia University. Teachers College. Bureau of Publications. 1944.

White. M. J. Laterality differences in perception: A review. Psychological Bulletin. 1969. 72. 387-405.

(Received for publication October 26. 1975.) 